\title{
Primary Neuroendocrine Carcinoma of Breast: An Uncommon Variant
}

\author{
Sunil V Jagtap ${ }^{1 *}$, Vidya Chandrashekhar Aher ${ }^{1}$, Suresh Jaywantrao Bhosale ${ }^{2}$, Atul Hulwan ${ }^{1}$ and Anup Nanagir Gosavi ${ }^{1}$ \\ ${ }^{1}$ Department of Pathology, Krishna Institute of Medical Sciences Deemed University (KIMSDU). Maharashtra, India \\ ${ }^{2}$ Department of Surgery, Krishna Institute of Medical Sciences Deemed University (KIMSDU). Maharashtra, India
}

\section{ABSTRACT}

Neuroendocrine carcinoma of breast ( NECB) is an aggressive variant of invasive mammary carcinoma. Very few cases of Primary NECB have been reported in the literature. Here we report a 50 year female presented with left breast lump measuring $15 \mathrm{x} 12 \mathrm{~cm}$. of gradual onset with left axillary lymphadenopathy. On fine needle aspiration cytology of breast and lymphnode were positive for malignant cells. On ultrasonography of abdomino- pelvis showed metastasis to liver, peri pancreatic and para aortic lymph nodes with minimal ascites. On histopathology reported as Primary NECB-Large cell type-left breast. We are presenting this case for its extreme rarity and aggressive clinical behaviour.

\section{Keywords: - Neuroendocrine Carcinoma of Breast, Endocrine Tumor, Breast Cancer, Histopathology}

\section{Introduction}

Neuroendocrine carcinoma of breast ( NECB) is an aggressive variant of invasive mammary carcinoma. It is extremely rare tumor accounting for 0.27 to $0.50 \%$ of histopathologically proven breast cancers ${ }^{[1,2]}$. Most neuroendocrine carcinomas are located in gastrointestinal tract and lung ${ }^{[3]}$. Only a limited number of studies on neuroendocrine breast have been reported in the literature and most of them are case studies.

\section{Case Report}

A 50 year old postmenopausal female presented with a single, large, hard, non-tender, gradually increasing, lump in the left breast of 8 months duration. At that time there was no other systemic disease was evident on clinical and other investigations. Patient delayed the initial treatment and came later with retraction of nipple and left axillary lymphadenopathy. She had no history of significant systemic disease. Her obstetrics history was G4P4D0L4 with menopause six years back. Her past and family history was non contributory. Radiological examination by ultrasonography of abdomen pelvis at second visit revealed metastasis to liver, peri pancreatic and para aortic lymph nodes with minimal ascites. Mammography showed heteroechoeic lesions with hypoechoic rims. Fine needle aspiration cytology left breast was positive for malignant cells. Modified radical mastectomy was performed .Histopathological examination revealed gross specimen of left breast measuring 20x18x9 $\mathrm{cm}$ and weighing $900 \mathrm{gms}$. It was covered with skin measuring $17 \times 15 \mathrm{~cm}$ (Fig.1). Tumour was fixed to skin with nipple areola retraction. Peau-d-orange was also noted. On cut section revealed a tumor measuring 15x12x6 cm (Fig.2). Tumor was grey white, firm to hard in consistency with pushing peripheral margins. On microscopy, multiple sections showed tumor composed of neoplastic cells, arranged in large sheets, alveolar pattern, solid nests separated by thin fibrous septae(Fig.3). Individual tumor cells were large, round, pleomorphic having salt and pepper chromatin and moderate eosinophilic granular cytoplasm which constituted more than $60 \%$ of tumor morphology(Fig.4). Focally invasive breast carcinoma is noted. Intervening stroma showed diffuse mononuclear cell infiltration and fibrosis. On histopathological examination diagnosed as NECB-Large cell type - left breast.

On Immunohistochemistry tumor showed $>50 \%$ tumor population showing positivity for Synaptophysin and chromogranin. Tumor was positive for ER and PR receptors. Our patient underwent left modified radical mastectomy and received chemotherapy and radiotherapy. On follow up, the patient is doing well.

\section{Discussion}

Primary NECB is an extremely rare specific subtype of breast malignancy . Most of the neuroendocrine tumors are located in the gastrointestinal tract and lung. The morphological features of primary NECB are similar to that of other sites. Many benign and malignant lesions of various organs show neuroendocrine differentiation ${ }^{[4]}$. Neuroendocrine differentiation in breast carcinoma shows morphological similarity to carcinoma of gastrointestinal tract, lung and other nonendocrine organs with endocrine differentiation and carcinoid tumor ${ }^{[5]}$. In breast carcinoma, 


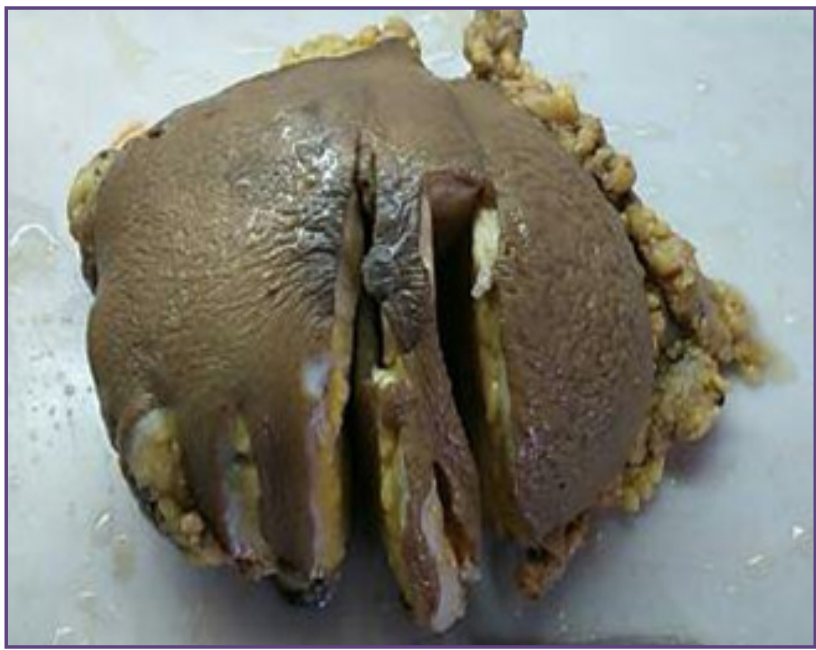

Fig. 1: Gross specimen of left breast- modified radical mastectomy.

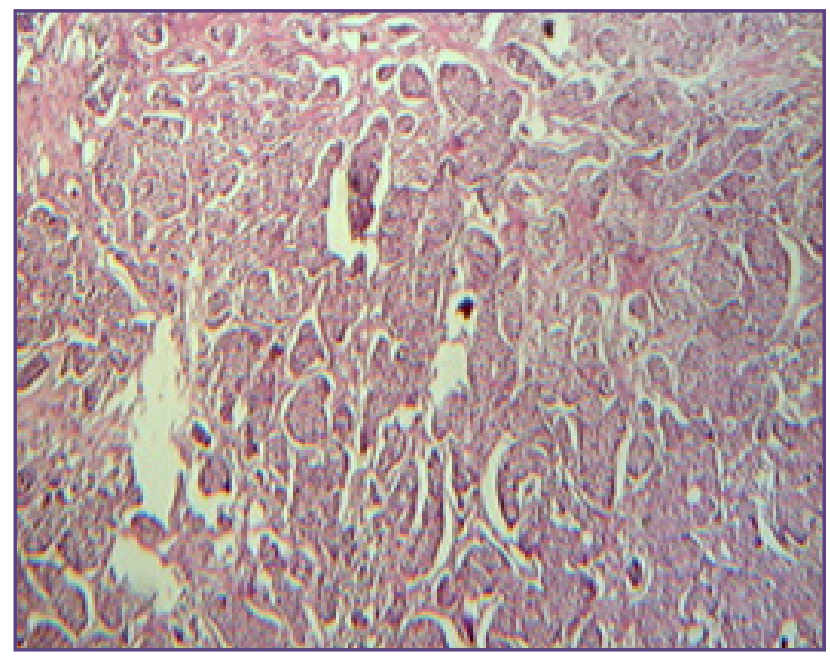

Fig. 3: photomicrograph showing breast tissue with tumor composed of neoplastic cells arranged in large sheets, alveolar pattern and solid nests separated by thin fibrovascular septa. H\&E stain 100X

not otherwise specified (NOS) focal neuroendocrine differentiation can be seen scattered singly or in groups ${ }^{[6]}$.

NECB used to be known as argyrophilic breast carcinoma, Carcinoid tumor or Endocrine carcinoma and now classified as breast carcinoma with neuroendocrine differentiation or Primary Neuroendocrine carcinoma ${ }^{[7,8]}$.

In $50 \%$ of breast tumors, scattered neuroendocrine cells can be detected. Neuroendocrine differentiation has been reported in both in situ and invasine breast carcinoma. It is observed that mucinous carcinoma of breast has the greatest association with neuroendocrine differentiation ${ }^{[8]}$. WHO in 2003 defined NECB as breast carcinoma with

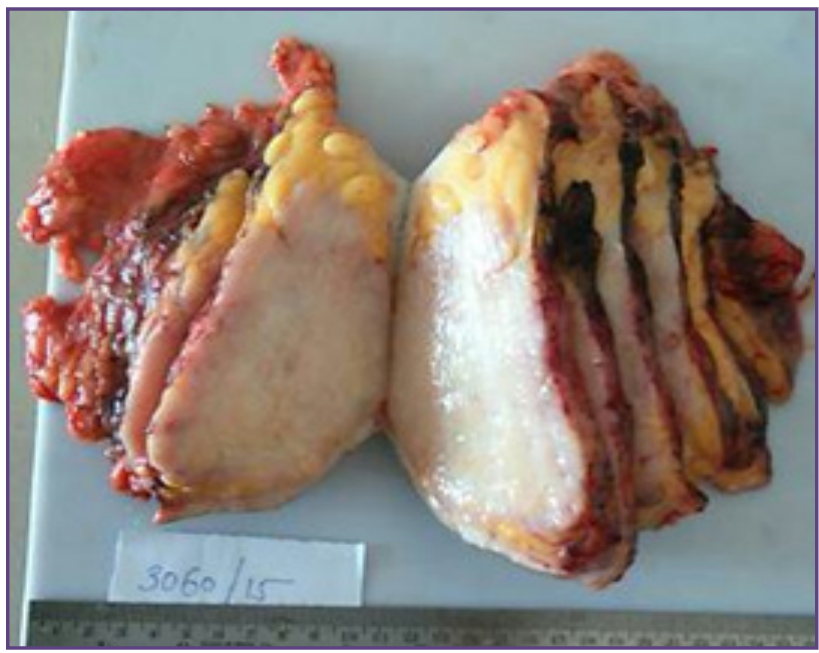

Fig. 2: left breast on cut open showing grey white, homogenous, firm to hard tumor with pushing borders m. $15 \times 12 \times 6 \mathrm{~cm}$.

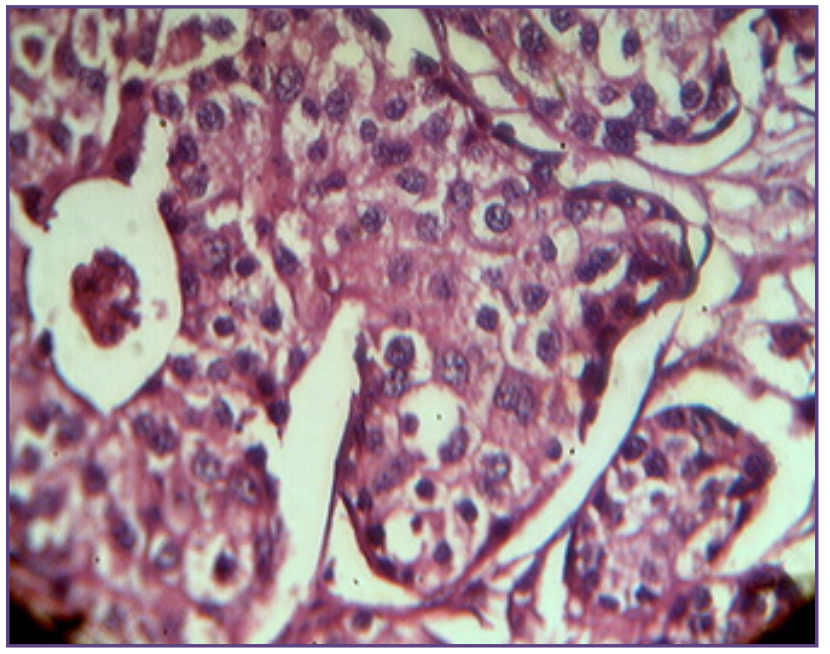

Fig. 4: Individual tumor cells are medium to large size, round to pleomorphic having salt and paper chromatin and moderate amount of eosinophilic granular cytoplasm. . H\&E stain 400X

more than $50 \%$ of the population of tumor expressing immunohistochemical staining for neuroendocrine markers for diagnosing primary NECB and the extra mammary sites should be excluded ${ }^{[7]}$.

Our patient was a 50 year old woman who presented with breast lump of 8 months duration which was gradually increasing in size with axillary node enlargement .Initially patient was not ready for surgical management but came at the clinic in late stage of disease. The mean age at diagnosis of the patient with NEC is 64 years. The mean tumor size is $3.2 \mathrm{~cm}$ for $\mathrm{NEC}{ }^{[6]}$. Where as in our case tumor was very large size as patient presented in late stage of disease. It 
is reported that about $43 \%$ of NEC cases presented with lymph node metastasis at the time of diagnosis [9]. In our case patient had significant axillary node involvement.

On histology, neuroendocrine carcinoma usually shows neoplastic cells arranged in alveolar pattern, solid sheets with tendency to produce peripheral palisidation. Depending on cell type and differentiation it is subtyped into- Solid neuroendocrine carcinoma, Oat cell carcinoma , Large cell neuroendocrine carcinoma ${ }^{[7,9,10]}$.

In large series by Sapino et al, described tumors in 5 subtypes; solid cohesive, alveolar, small cell, solid papillary, cellular mucinous ${ }^{[6]}$.Our case shows predominant solid, alveolar and nest pattern having large cells with focal areas of mucin production. Few cases reported in literature of primary breast NEC of large cell type by Kim JW et al ${ }^{[2]}$, Stita W et al ${ }^{[10]}$, Hanna MY et al ${ }^{[1]}$, Wei B et al. ${ }^{[12]}$. Overall survival of patients with NEC is determined significantly by tumour size, lymph node status, and proliferation rate. Clinical outcomes reported in literature showed $15 \%$ of local recurrence by 5 years, with median recurrence free time of 177 months and 34\% risk for distant recurrence within 5 years ${ }^{[12]}$.

\section{Conclusion}

Primary NECB is an unusual and aggressive carcinoma of breast. We are presenting this case for its extreme rarity and with advanced clinical behaviour. The treatment modality is not different from those conventional breast malignancies, however chromogranin production in neuroendocrine carcinoma has clinical and genetic implications owing to the biochemical analogy between granin and breast cancer (BRCA) protein. Hence, targeted gene therapy may be a future treatment strategy.

\section{References}

1. Zhang Y, Chen Z, Bao Y, Du Z, Li Q, Zhao Y, et al. Invasive neuroendocrine carcinoma of the breast: a prognostic research of 107 Chinese patients. Neoplasma 2013;60:215-22.
2. Kim JW, Woo OH, Cho KR, Seo BK, Yong HS, Kim A, et al. Primary large cell neuroendocrine carcinoma of the breast: radiologic and pathologic findings. J Korean Med Sci 2008;23:1118-20.

3. Modlin IM, Lye K D, Kidd M. A five decade analysis of 13,715 carcinoid tumors. Cancer.2003;97:934-959.

4. David O, Bhattacharjee M. Diffuse neuroendocrine differentiation in a morphologically composite mammary infiltrating ductal carcinoma:a case report and review of literature. Arch Pathol Lab Med.2003;127(3):e131-4.

5. Ozbilim G, Kilicarslan B, Tezer E, Buyukkece A, Ustum M, Karaveli $\mathrm{S}$ et al. Breast carcinoma showing neuroendocrine differentiation characterized with ectopic hormone production (2 case report). Turk J Med Sci 2000;30:609-13.

6. Sapino A, Righi F, Cassoni P, Papitti M, Gugliotta $\mathrm{P}$, Bussolati G. Expression of the neuroendocrine phenomenon in carcinoma of the breast. SeminDiagnPathol 2000; 17:127-37.

7. Ellis IO, Schnitt SJ, Saste-Garau X. Invasive breast carcinoma. In: Tavassoli FA, Devilee P, editors. World Health Organization classification of tumours. Pathology and genetics of the tumours of breast and female genital organs. Lyon: IARC press;2003:13-59.

8. Tovosoli FA, Pathology of the breast. 1st ed. Norwalk: Connecticut. Appleton and Lange;2000.

9. Wang Jun, Bing Wei, Constance TA, et al. Invasive neuroendocrine carcinoma of breast: a population based study from the surveillance, epidemiology and end result (SEER) database, BMC cancer.2014;14:147.

10. Stita W, Trabelsi A, Gharbi O, Mokni M, Korbi S. Primary solid neuroendocrine carcinoma of the breast Can J Surg 2009;52:E289-E290.

11. Hanna MY, Leung E, Rogers C, Pilgrim S. Primary large-cell neuroendocrine tumor of the breast. Breast J. 2013;19:204-206.

12. Wei B, Ding T, Xing Y, Wei W, Tian Z, Tang F, et al. Invasive neuroendocrine carcinoma of the breast: A distinctive subtype of aggressive mammary carcinoma. Cancer. 2010;116:4463-73.

*Corresponding author:

Dr Sunil Vitthalrao Jagtap, Gadkari-mala, Vadgaon haveli-415110, Maharashtra, India.

Email: drsvjagtap@gmail.com

Date of Submission : 10.06.2016

Date of Acceptance : 19.01.2017

Financial or other Competing Interests: None.
Date of Publication : 28.03.2017 